\title{
Partition function of nearest neighbour Ising models: Some new insights $^{\dagger}$
}

\author{
G NANDHINI and M V SANGARANARAYANAN* \\ Department of Chemistry, Indian Institute of Technology Madras, Chennai 600036 \\ e-mail: sangara@iitm.ac.in
}

\begin{abstract}
The partition function for one-dimensional nearest neighbour Ising models is estimated by summing all the energy terms in the Hamiltonian for $\mathrm{N}$ sites. The algebraic expression for the partition function is then employed to deduce the eigenvalues of the basic $2 \times 2$ matrix and the corresponding Hermitian Toeplitz matrix is derived using the Discrete Fourier Transform. A new recurrence relation pertaining to the partition function for two-dimensional Ising models in zero magnetic field is also proposed.
\end{abstract}

Keywords. Ising model; partition function, Toeplitz matrix; recurrence relation; Discrete Fourier Transform.

\section{Introduction}

The analysis of nearest neighbour Ising models plays a crucial role in diverse contexts such as orderdisorder transitions, ${ }^{1}$ adsorption at electrochemical interfaces, ${ }^{2}$ protein folding, ${ }^{3}$ etc. While the formulation of the partition function pertaining to onedimensional nearest neighbour Ising models is pedagogical and straight-forward, ${ }^{4}$ the same is not true for the two-dimensional Ising models. The celebrated solution of Onsager ${ }^{5}$ for the two-dimensional Ising model at $H=0$ led to the detailed analysis of critical temperatures and critical exponents. However, several approximate treatments for the analysis of two-dimensional Ising models employing BraggWilliams approximation, ${ }^{6}$ Bethe ansatz, ${ }^{7}$ series expansions, ${ }^{8}$ etc. are available in addition to the Monte Carlo simulations. ${ }^{9}$ A significant accomplishment regarding the exact solution of the two-dimensional Ising models consists in the application of the renormalization group techniques ${ }^{10}$ for the study of critical phenomena. Nevertheless, the exact partition function for the two-dimensional Ising model in the presence of the external magnetic field $(H \neq 0)$ leading to the critical properties is still lacking. A plausible strategy to obtain new insights regarding the analysis of two-dimensional Ising models is to formulate new procedures even for one-dimensional Ising models in anticipation that this new methodo-

\footnotetext{
${ }^{\dagger}$ Dedicated to the memory of the late Professor S K Rangarajan *For correspondence
}

logy may lead to the exact solution of the twodimensional Ising models in presence of an external magnetic field.

It is of interest to point out here that the partition function for one-dimensional nearest neighbour Ising models for $N$ sites is customarily derived by deducing the eigenvalues of a $2 \times 2$ Matrix and subsequently generalized to the thermodynamic limit $N \rightarrow \infty$.

The objectives of the present Communication are (i) to report the partition function of one-dimensional nearest neighbour Ising models by summation of all the energy terms in the Hamiltonian for small values of $N$; (ii) to re-construct the matrix from the known eigenvalues and (iii) to postulate a recurrence relation between the partition function for $N$ and $2 N$ sites.

\section{Partition function of one-dimensional nearest neighbour Ising models}

The Hamiltonian for the one-dimensional Ising model is

$$
H_{T}=-J \sum_{i=1}^{N} \sigma_{i} \sigma_{i+1}-H \sum_{i=1}^{N} \sigma_{i},
$$

where $J$ denotes the nearest neighbour interaction energy and $H$ denotes the external magnetic field. It is customary to construct a basic $2 \times 2$ matrix consisting of the matrix elements which takes into account, various arrangements of the spin variables 
$(+1,+1 ;-1,+1 ;+1,-1$ and $-1,-1)$. The canonical partition function then is deduced as ${ }^{4}$

$$
Q_{N}=\left(\lambda_{1}^{N}+\lambda_{2}^{N}\right)
$$

where $\lambda_{1}$ and $\lambda_{2}$ denote the eigenvalues:

$$
\begin{aligned}
& \lambda_{1}=e^{J / k T}\left[\cosh \left(\frac{H}{k T}\right)+\sqrt{\sinh ^{2}\left(\frac{H}{k T}\right)+e^{-4 J / k T}}\right] \\
& \lambda_{2}=e^{J / k T}\left[\cosh \left(\frac{H}{k T}\right)-\sqrt{\sinh ^{2}\left(\frac{H}{k T}\right)+e^{-4 J / k T}}\right]
\end{aligned}
$$

which arise from the matrix

$$
Q=\left(\begin{array}{cc}
e^{1 / k T(J+H)} & e^{-J / k T} \\
e^{-J / k T} & e^{1 / k T(J-H)}
\end{array}\right)
$$

One of the two eigenvalues being the most dominant, estimation of the partition function in the thermodynamic limit and extraction of the internal energy, specific heat, etc. becomes straight-forward.

\subsection{Partition function from the summation of the energy terms}

In stead of the above method involving the formulation of the matrix and computing the partition function from the eigenvalues, we investigate here, the consequences of summation of the energy terms directly. The partition function obtained by summing the energy terms for different values of $N$ (3 and 6;4 and 8) are as follows:

$$
\begin{gathered}
Q_{3}=2 e^{3 J / k T} \cosh \left(\frac{3 H}{k T}\right)+6 e^{-J / k T} \cosh \left(\frac{H}{k T}\right) \\
Q_{4}=2 e^{-4 J / k T} \cosh \left(\frac{4 H}{k T}\right) \\
+8 \cosh \left(\frac{2 H}{k T}\right)+\left(4+2 e^{-4 J / k T}\right) \\
Q_{6}=2 e^{6 J / k T} \cosh \left(\frac{6 H}{k T}\right)+12 e^{2 J / k T} \cosh \left(\frac{4 H}{k T}\right) \\
+2\left(6 e^{2 J J k T}+9 e^{-2 J J k T}\right) \cosh \left(\frac{2 H}{k T}\right)
\end{gathered}
$$

$$
Q_{8}=2 e^{8 J / k T} \cosh \left(\frac{8 H}{k T}\right)+16 e^{4 J / k T} \cosh \left(\frac{6 H}{k T}\right)
$$

$$
\begin{aligned}
& +2\left(8 e^{4 J / k T}+20\right) \cosh \left(\frac{4 H}{k T}\right) \\
& +2\left(8 e^{4 J / k T}+32+16 e^{-4 J / k T}\right) \cosh \left(\frac{2 H}{k T}\right) \\
& +\left(8 e^{4 J / k T}+36+24 e^{-4 J / k T}+2 e^{-8 J / k T}\right) .
\end{aligned}
$$

Equations (5) to (8) were deduced by listing all the energy terms of (2) using MS EXCEL $\left(2^{N}\right.$ terms arise for a lattice of $N$ sites). Although the procedure is valid for any value of $N$, we restricted the enumeration to a small number of sites so as to avoid algebraic complexity as well as for obtaining a recurrence relation for the partition function.

\subsection{Eigenvalues from the partition function}

As an illustration, consider the partition function obtained by summation of the energy terms in the Hamiltonian (1) for $N=4$ :

$$
\begin{aligned}
Q_{4}= & 2 e^{4 J / k T} \cosh \left(\frac{4 H}{k T}\right)+8 \cosh \left(\frac{2 H}{k T}\right)+\left(4+2 e^{-4 J / k T}\right) \\
= & 2 e^{4 J / k T}\left[\cosh ^{4}\left(\frac{H}{k T}\right)+\sinh ^{4}\left(\frac{H}{k T}\right)\right] \\
& +12 e^{4 J J k T} \cosh ^{2}\left(\frac{H}{k T}\right) \sinh ^{2}\left(\frac{H}{k T}\right) \\
& +8\left[\cosh 2\left(\frac{H}{k T}\right)+\sinh ^{2}\left(\frac{H}{k T}\right)\right]+4+2 e^{-4 J / k T} \\
= & {\left[\left(e^{J / k T} \cosh \left(\frac{H}{k T}\right)+\sqrt{e^{2 J / k T} \sinh ^{2}\left(\frac{H}{k T}\right)+e^{-2 J J k T}}\right)^{4}\right.} \\
+ & \left.+\left(e^{J / k T} \cosh \left(\frac{H}{k T}\right)-\sqrt{e^{2 J / k T} \sinh ^{2}\left(\frac{H}{k T}\right)+e^{-2 J / k T}}\right)^{4}\right] .
\end{aligned}
$$


As can be seen from (2), $Q_{4}$ can also be represented as

$$
Q_{4}=\left(\lambda_{1}^{4}+\lambda_{2}^{4}\right)
$$

where $\lambda_{1}$ and $\lambda_{2}$ denote the eigenvalues of the matrix corresponding to the one-dimensional Ising model.

A comparison of (9) and (10) leads to the eigenvalues as given by (3).

\subsection{Recurrence relation}

In view of the availability of convenient expressions for $Q$, it is imperative to verify whether there exists any pattern among the partition function for different values of $N$. Using well-known identities involving trigonometric functions, ${ }^{11}$ it can be shown that

$$
Q_{2 N}=Q_{N}^{2}-2^{N+1} \sinh ^{N}\left(\frac{2 J}{k T}\right)
$$

for all values of $N$. The above eqn thus enables predicting the partition function for a lattice of $2 N$ sites from the value pertaining to $N$ sites.

\subsection{Inverse problem of constructing the matrix from the eigenvalues}

In the present approach, while the partition function and the eigenvalues have been obtained, the underlying matrix has not yet been derived. Obviously, the matrix is not unique for a given eigenspectrum; however, the construction of such matrices may provide an insight into the solution of higher dimensional Ising models. To obtain the matrix, we apply a procedure demonstrated in the context of signal processing ${ }^{12}$ employing the Discrete Fourier Transform. For a given set of eigenvalues $\left(\lambda_{0}, \lambda_{1}, \ldots, \lambda_{n-1}\right)$, the parameters $q_{k}$ are defined as:

$$
\begin{gathered}
q_{k}=\frac{1}{n} \sum_{i=0}^{n-1} \lambda_{i} \cos \frac{\pi}{m}(2 i+1) k, \\
k=0,1, \ldots, n-1 .
\end{gathered}
$$

The associated matrix $[q]$ consisting of elements $q_{k}$ is given below: (cf. Appendix A):

$$
\begin{aligned}
& {[q]=} \\
& {\left[\begin{array}{ccccc|ccccc}
q_{0} & q_{1} & \ldots & & q_{n-1} & 0 & -q_{n-1} & \ldots & -q_{2} & -q_{1} \\
q_{1} & q_{0} & \ldots & & q_{n-2} & q_{n-1} & 0 & \ldots & & -q_{2} \\
\vdots & \vdots & \ddots & & \vdots & \vdots & \vdots & \ddots & \vdots & \vdots \\
q_{n-1} & q_{n-2} & \ldots & & q_{0} & q_{1} & q_{2} & \ldots & q_{n-1} & 0 \\
\hline 0 & q_{n-1} & \ldots & q_{2} & q_{1} & q_{0} & q_{1} & \ldots & q_{n-1} \\
-q_{n-1} & 0 & \ldots & & q_{2} & q_{1} & q_{0} & \ldots & q_{n-2} \\
\vdots & \vdots & \ddots & \vdots & \vdots & \vdots & \vdots & \ddots & \vdots \\
-q_{1} & -q_{2} & \ldots & -q_{n-1} & 0 & q_{n-1} & q_{n-2} & \ldots & & q_{0}
\end{array}\right]}
\end{aligned}
$$

while the desired matrix constructed from the above is as follows, ${ }^{12}$

$$
C=\left[\begin{array}{ccccc}
q_{0} & q_{1}-i q_{n-1} & \ldots & & q_{n-1}-i q_{1} \\
q_{1}+i q_{n-1} & q_{0} & \ldots & \ddots & q_{n-2}-i q_{2} \\
\vdots & \vdots & \ddots & & \\
q_{n-1}-i q_{1} & q_{n-2}-i q_{2} & \ldots & & q_{0}
\end{array}\right] .
$$

In the case of the one-dimensional Ising model, the above procedure to construct the matrix leads to the values of $q_{k}(k=0,1)$ as

$$
\begin{aligned}
& q_{0}=e^{J / k T} \cosh \left(\frac{H}{k T}\right) \\
& q_{1}=e^{J / k T} \sqrt{\frac{\sinh ^{2}\left(\frac{H}{k T}\right)+e^{-4 J / k T}}{2}} .
\end{aligned}
$$

The corresponding matrix turns out to be

$$
C=e^{J / k T}
$$

$\left[\begin{array}{l}\cosh \left(\frac{H}{k T}\right) \\ (1+i) \sqrt{\frac{\sinh ^{2}\left(\frac{H}{k T}\right)+e^{-4 J / k T}}{2}} \cosh \left(\frac{H}{k T}\right)\end{array}\right]$

It is of interest to note that the above Hermitian Toeplitz matrix is entirely different from the hith- 
erto-known matrix (4) for one-dimensional nearest and neighbour Ising models.

\section{Two-dimensional nearest neighbour Ising models}

It will be of interest to verify whether this methodology will be applicable to two-dimensional Ising model in presence of the external magnetic field. The summation of energy terms for two-dimensional Ising model for $H \neq 0$ has earlier been carried out by us $^{13}$ and the formulation of the matrix using the Discrete Fourier Transform is under progress. However, here we make use of the Onsager's exact solution for $H=0$ and demonstrate the validity of the recurrence relation (11) for two-dimensions also. Such recurrence relations can enable extrapolation of Monte Carlo and molecular dynamics simulation results for larger system sizes.

The canonical partition function for $H=0$ arising from Onsager's exact solution is given by ${ }^{5}$

$$
Q_{H=0}=\left[2 \cosh \left(\frac{2 J}{k T}\right) e^{I}\right]^{N},
$$

where

$$
I=\frac{1}{2 \pi} \int_{0}^{\pi} \frac{1}{2} \log \left(1+\sqrt{1-k_{1}^{2} \sin ^{2} \phi}\right) \mathrm{d} \phi
$$

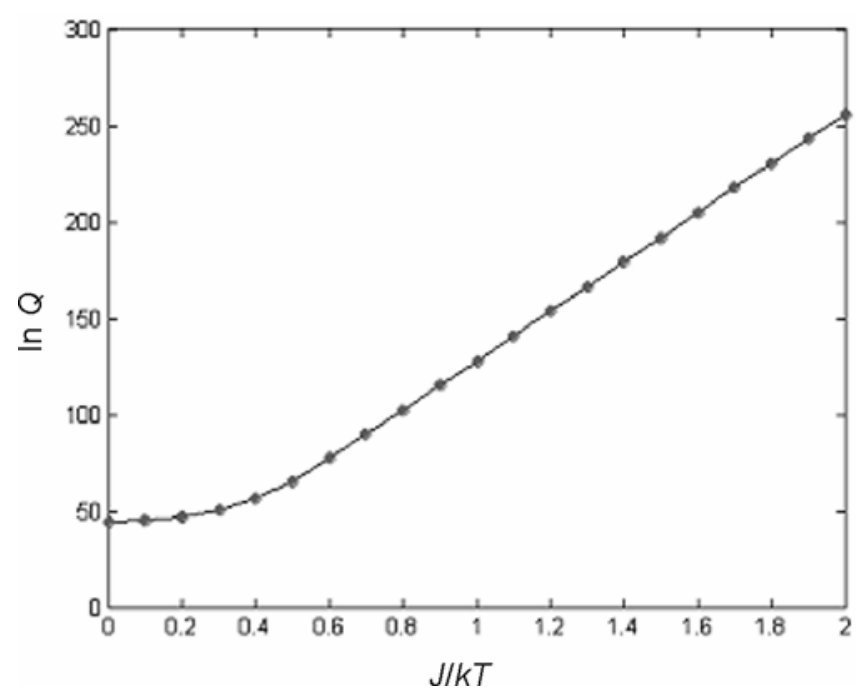

Figure 1. The comparison of the partition function values from Onsager's exact solution (16) with the values predicted by the recurrence relation (11) The points denote the values from (11) for $Q_{64}$ employing the values of $Q_{16}$ from Onsager's exact solution (16) while the line is the predicted values from (16) employing $N=64$.

$$
k_{1}=\frac{2 \sinh (2 J / k T)}{\cosh ^{2}(2 J / k T)} .
$$

Employing the above equations, it is possible to estimate $Q$ for different values of $N$. Since our essential focus has been on the square lattice, we first substitute $N=16$ in (11) and calculate $Q$ for $N=32$; subsequently, the value of $Q$ for $N=64$ is evaluated from (11). This estimated value is compared with $Q$ values arising from (16) for $N=64$ (figure 1). As can be seen from figure 1, the agreement is entirely convincing, suggesting that the recurrence relation (11) is valid for two-dimensional nearest neighbour Ising model too, when $H=0$. It is interesting to note that the values for $N=16$ was employed as the input to predict the values for $N=64$.

\section{Summary}

An alternate method for estimating the partition function of one-dimensional nearest neighbour Ising model is proposed. A new recurrence relation is proposed for the partition function for $N$ sites. A Hermitian Toeplitz matrix is constructed from the eigenvalues, using the Discrete Fourier Transformation. The significance of this method for twodimensional Ising models when $H=0$ is indicated.

\section{Acknowledgement}

This work was supported by the Department of Science and Technology (DST), Government of India, New Delhi.

\section{Appendix A}

In this Appendix, the steps leading to the construction of the matrix (14) from the eigenvalues spectrum is indicated. The analysis is identical with that of $^{12}$ employed in the context of signal processing. A negacyclic real symmetric matrix of order $\mathrm{n}$ can be obtained from a set of $\mathrm{n}$ distinct eigenvalues by use of the inverse DFT,

$$
q_{k}=\frac{1}{n} \sum_{i=0}^{n-1} \lambda_{i} \cos \frac{\pi}{m}(2 i+1) k, \quad k=0,1, \ldots, n-1 .
$$


As an illustration, for an eigen spectrum consisting of 3 eigenvalues, $n=3$ and hence $m=2 n=6$, a $6 \times 6$ matrix is first constructed.

$$
\left[q_{e x}\right]=\left[\begin{array}{ccc|ccc}
q_{0} & q_{1} & q_{2} & 0 & -q_{2} & -q_{1} \\
q_{1} & q_{0} & q_{1} & q_{2} & 0 & -q_{2} \\
q_{2} & q_{1} & q_{0} & q_{1} & q_{2} & 0 \\
\hline 0 & q_{2} & q_{1} & q_{0} & q_{1} & q_{2} \\
-q_{2} & 0 & q_{2} & q_{1} & q_{0} & q_{1} \\
-q_{1} & -q_{2} & 0 & q_{2} & q_{1} & q_{0}
\end{array}\right]
$$

The above matrix can be re-written in the form

$$
\left[\begin{array}{cc}
{[A]} & -[B] \\
{[B]} & {[A]}
\end{array}\right]
$$

where

$$
[A]=\left[\begin{array}{lll}
q_{0} & q_{1} & q_{2} \\
q_{1} & q_{0} & q_{1} \\
q_{2} & q_{1} & q_{0}
\end{array}\right],[B]=\left[\begin{array}{ccc}
0 & q_{2} & q_{1} \\
-q_{2} & 0 & q_{2} \\
-q_{1} & -q_{2} & 0
\end{array}\right] .
$$

If we assume the matrix elements of $A$ as $a_{11}, a_{12}$, $a_{13}$, etc. and that of the matrix $B$ as $b_{11}, b_{12}, b_{13}, b_{21}$, $b_{22}$, etc. it follows that $a_{11}=q_{0}, a_{12}=q_{1}, \ldots, a_{33}=q_{0}$ and $b_{11}=0, b_{12}=q_{2}, \ldots, b_{33}=0$, the desired matrix $C$ with the elements $c_{11}, c_{12}$, etc. are obtained as $c_{11}=a_{11}-i b_{11}, \quad c_{12}=a_{12}-i b_{12}, \ldots, c_{33}=a_{33}-i b_{33}$, yielding

$$
C=\left[\begin{array}{ccc}
q_{0} & q_{1}-i q_{2} & q_{2}-i q_{1} \\
q_{1}+i q_{2} & q_{0} & q_{1}-i q_{2} \\
q_{2}+i q_{1} & q_{1}+i q_{2} & q_{0}
\end{array}\right] .
$$

where $i=(-1)^{1 / 2}$. This procedure was employed for deducing the matrix (15) pertaining to the onedimensional Ising model.

\section{References}

1. Bonaccorsi E, Merlino S, Pasero M and Macedonio G 2001 Phys. Chem. Miner. 28509

2. Basha C A and Sangaranarayanan M V 1989 J. Elec. Anal. Chem. 261431

3. Lenz P, Zagrovic B, Shapiro J and Pande V S $2004 J$. Chem. Phys. 1206769

4. Huang K 1963 Statistical mechanics (New York: John Wiley) 1st edn

5. Onsager L 1944 Phys. Rev. 65117

6. Bragg W L and Williams E J 1934 Proc. Roy. Soc (London) A145 699

7. Bethe H A 1935 Proc. Roy. Soc. (London) A150 552

8. Domb C, In Domb C and Green M S (eds) 1974 Phase transitions and critical phenomena (London: Academic Press) vol 3, pp. 357-484

9. David P, Landau and Kurt Binder 2000 A guide to Monte Carlo simulations in statistical physics (Cambridge University Press) p. 71

10. Wilson K G 1971 Phys. Rev. B4 3174

11. Gradshteyn IS and Ryzhik IM 1996 Table of integrals, series and products (Academic Press) p. 31

12. Noor F and Morgera S D 1992 IEEE Trans. Signal Process. 402093

13. Nandhini G and Sangaranarayanan M V archives: http://uk.arxiv.org/ftp/arxiv/papers/0710/0710.3688.pdf 